\title{
Metastatic Melanoma of the Gallbladder: An Unusual Image finding in an Asymptomatic Patient Diagnosed with FDG PET/CT; Case Report and Review of Literature
}

\author{
Taalab KM and *Mohamed Fathy Ragab \\ PET/CT Unit, Techno Scan, Egypt
}

Submission: April 02, 2017; Published: April 13, 2017

"Correspondence Address: Mohamed Fathy Ragab, PET/CT Unit, Techno Scan, Egypt

\begin{abstract}
Introduction: Gallbladder metastasis of malignant melanoma is rare and generally originates from skin melanoma. We report a case of gallbladder metastasis from a malignant melanoma of the uvea that is diagnosed with FDG PET/CT.

Case presentation and Image findings: A 45-year-old man diagnosed with malignant melanoma of the right uvea seven years ago developed recently a scalp nodule that was proved pathologically to be metastatic melanoma. He underwent systemic screening PET/CT and increased FDG uptake was detected in the scalp lesion, right cervical lymph nodes, brain, lungs, right adrenal gland, right anterior chest wall, bone and gallbladder.

Discussion: Only $2 \%$ to $4 \%$ of patients with melanoma will be diagnosed with gastrointestinal metastasis during the course of their disease. The most common sites of gastrointestinal metastases from melanoma include the small bowel (35\%-67\%), colon (9\%-15\%) and stomach (5\%-7\%), with 18\% survival at five years. In the literature, cutaneous melanoma is described as the origin of most metastatic gallbladder melanomas. Metastatic melanoma to the gallbladder is extremely rare and it is associated with a very poor prognosis.

Conclusion: In general, melanoma is FDG-avid and the sensitivity and specificity of distant metastasis measured by FDG PET/CT is reported to be $92 \%$ and $90 \%$, respectively. Accordingly, FDG PET/CT is considered a powerful imaging modality that can afford a crucial role in detection of rare metastatic melanoma lesions and provides more accurate staging information to the therapists' team.
\end{abstract}

\section{Introduction}

Malignant melanomas can metastasize to any organ; the most common sites of distant metastases are the lung (70-87\%), liver (54-77\%), and skin (50-75\%). Within the gastrointestinal tract, only $(2 \%-4 \%)$ of patients with melanoma will be diagnosed with gastrointestinal metastasis during the course of their disease. Melanomas metastasize most commonly to the small intestine (approximately 67\%), colon (9\%-15\%), stomach $(5 \%-7 \%)$ and rarely to the gallbladder. Autopsy studies have shown that gallbladder metastases are found in $15 \%$ of patients with metastatic melanoma of the gastrointestinal tract, but are reported extremely rarely during the lifetime of the patient; only about 40 such occurrences have been reported in the Englishlanguage literature; because involvement of the gallbladder seldom produces symptoms. Metastases of the biliary tract are rare and among metastatic gallbladder tumors, melanomas are the most common origin of malignancy, accounting for $50-60 \%$ of all reported gallbladder metastases [1-6].
FDG PET/CT; being a whole body imaging modality; has the advantage of multi-systemic screening with high sensitivity and specificity that can help in better detection of such lesions with more confidence; even in asymptomatic patients; which is expected to narrow the discrepancy between these autopsy findings and clinical detection rates; hence, provides more accurate staging data which will help therapists in more effective treatment planning. We report a case of metastatic malignant melanoma of the gallbladder in a 45-year-old man with a widely disseminated disease. According to the best of our knowledge, only few cases of metastatic malignant melanoma of GB were reported before cholecystectomy or before autopsy.

\section{Case Presentation and Image Findings}

A 45y/o man was diagnosed with right uveal malignant melanoma approximately 7 years ago, underwent surgical resection with favorable outcome. Four years later, he developed right conjunctiva recurrence that was managed through 


\section{Cancer Therapy \& Oncology International Journal}

surgery and cryo-therapy followed by immune-therapy with favorable response. Few months ago, he developed left parietal swelling and biopsy revealed metastatic malignant melanoma at histopathology. He also noticed a right sided anterior chest wall swelling only few weeks prior to the FDG PET/CT scan [715]. For the aforementioned patient we performed a true whole body PET/CT scan approximately one hour after intra-venous injection of $407 \mathrm{MBq}$ of $18 \mathrm{~F}-\mathrm{FDG}$ (patient weighted $85 \mathrm{~kg}$ ) using a 64 multi-slice GE Discovery VCT and a low dose CT was done simultaneously and used for attenuation correction and anatomical localization. Complementary contrast enhanced CT scan was also carried out for better anatomical delineation and diagnosis.
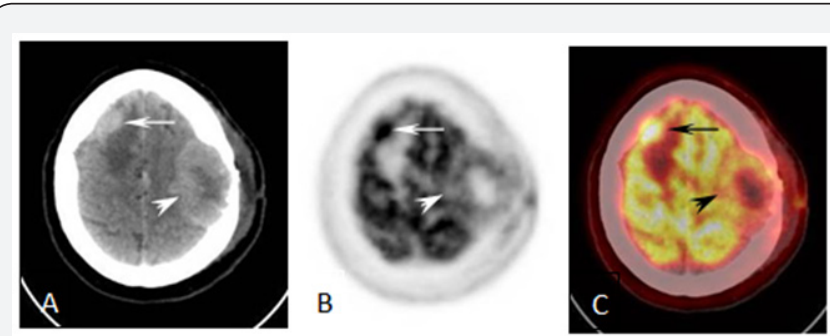

Figure 1: axial CE CT (A), PET (B) \& fused PET/CT (C) images showing glucose avid left parietal SOL with intra- \& extra-cranial components (arrow heads). Note another glucose avid right parietal brain SOL surrounded by glucose starved area of perilesional brain edema (arrows).

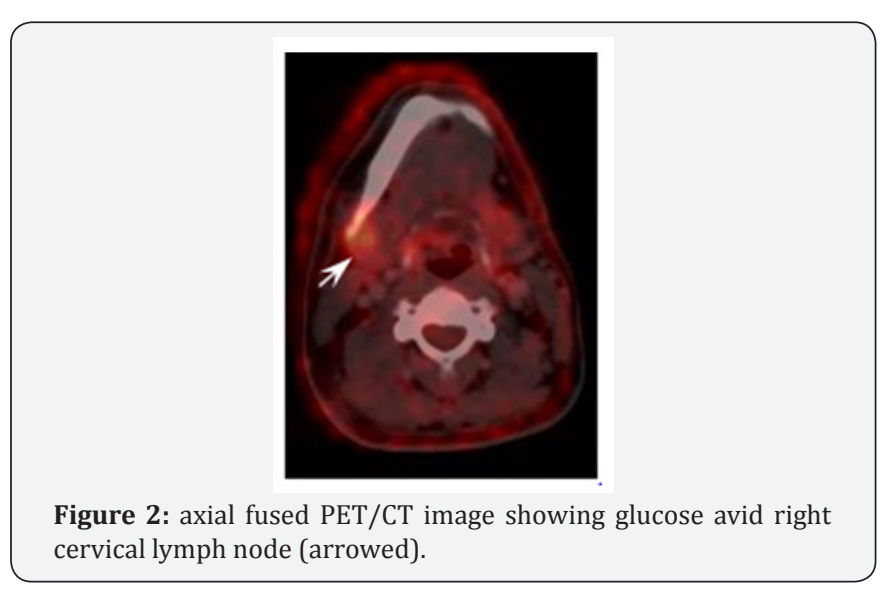

Maximum variant of standard uptake value (SUV); for semiquantitative analysis; was done for the selected regions of interest (ROI.s) and the normal threshold was assumed to be $<2.5$. The study revealed focal increased FDG uptake involving the left parietal region with intra- and extra-cranial components, right anterior parietal brain space occupying lesion surrounded by FDG starved peri-lesional brain edema and enlarged right cervical lymph node with SUV of 11,14 and 5 respectively (Figures 1 \& 2). While in the chest region, focal increased FDG uptake was noted at multiple bilateral pulmonary nodules and right sided anterior chest wall soft tissue mass that had intraand extra-thoracic components with SUV of 7 and 11 respectively (Figure 3). In the abdomen, focal increased FDG utilization was noted in a right adrenal mass with SUV 13 (Figure 4) while the liver appeared free from any foci of dense FDG uptake. Also, multiple osteolytic lesions were noted involving C6 vertebral body, L5 vertebral body, sacrum, both iliac bones, left femoral head \& neck, left pubic and ischeal bones; eliciting increased FDG fixation with SUV reaching up to 12 (Figure 5). The study also revealed a focus of dense FDG uptake with SUV 4 correlated to a gallbladder fundal soft tissue nodule measuring $1.3 \times 1.2$ $\mathrm{cm}$ in maximum axial dimensions which was better visualized in the complementary post-contrast CT series (Figure 6). High vascularity of the gallbladder nodule was also ascertained via complementary contrast-enhanced CT which showed avid homogeneous pattern of contrast enhancement.

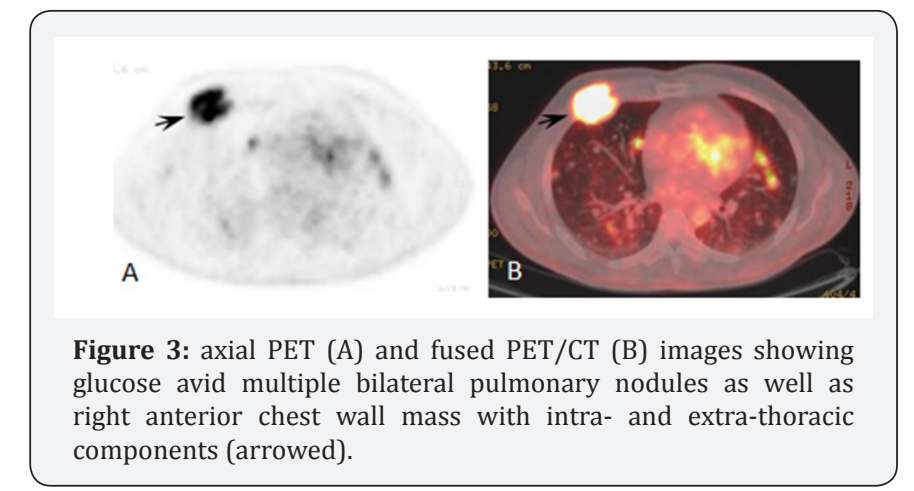

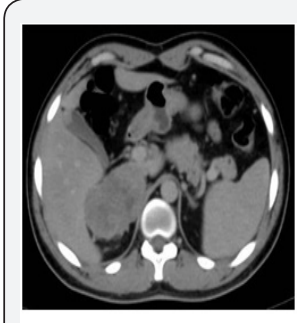

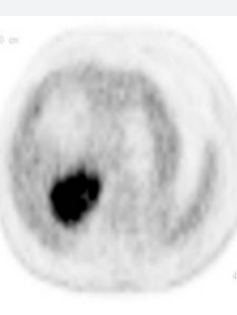

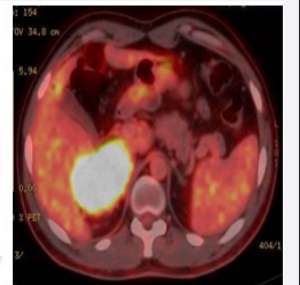

Figure 4: axial CE CT (A), PET (B) and fused PET/CT (C) images showing glucose avid right adrenal mass.

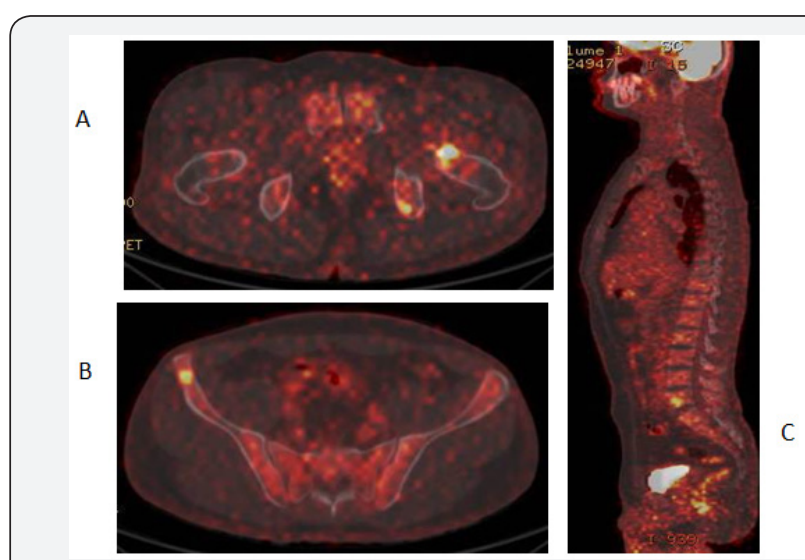

Figure 5: axial fused PET/CT (A \& B) and sagittal (C) images showing glucose avid multiple osseous deposits. 


\section{Cancer Therapy \& Oncology International Journal}

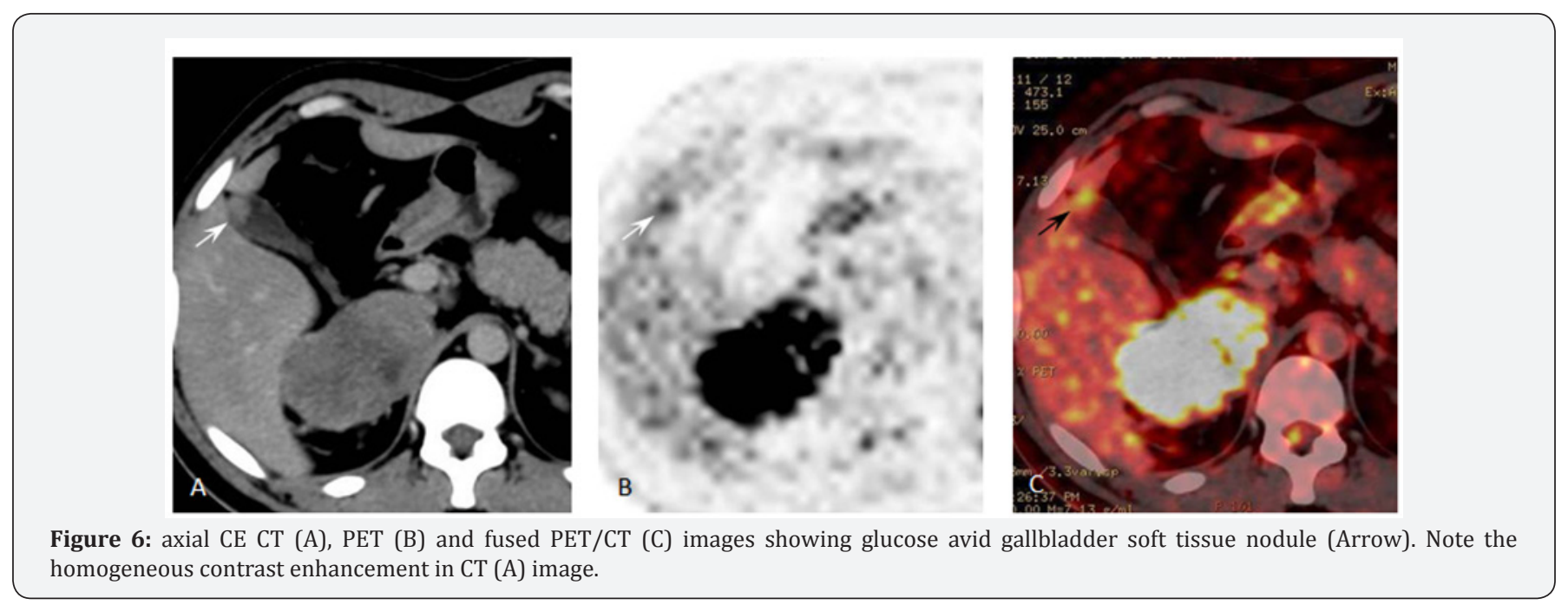

\section{Discussion}

Of all the tumors that can metastasize to the gallbladder, melanoma is by far the most common, accounting for more than $50 \%$ of gallbladder metastases. It is unknown why melanoma tends to metastasize to the gallbladder, but the reason may be related to how frequently melanoma spreads hematogenously. Metastases of malignant melanoma to the gallbladder and biliary tree are uncommon and usually are clinically asymptomatic. The most common sites of distant metastases are the skin, lungs, liver and brain. According to autopsy results, such asymptomatic metastases to the gallbladder and bile ducts occur in about $15 \%$ of patients with GIT metastatic melanoma. Despite these statistical data, it is rare for metastatic gallbladder melanoma to be present with symptoms during the patient's life time, as demonstrated by the discrepancy in published case reports and the rate of detection at autopsy [16-22].

The most frequent clinical manifestation in rare symptomatic cases is right upper quadrant or epigastric pain and tenderness that mimics cholecystitis. This manifestation is thought to be caused by obstruction of the cystic duct by the tumor mass. Melanoma of the gallbladder that manifests as acute cholecystitis is uncommon; approximately 15 cases are reported in the English-language literature. Extension into the biliary tree, jaundice, biliary fistulae, and hemobilia also have been described. Other reported symptoms include weight loss, food intolerance, nausea, vomiting, and diarrhea. Unlike gallbladder cancer, melanoma of the gallbladder is not associated with cholelithiasis. Also, hematobilia and biliary fistulae may develop. Most primary melanomas that metastasize to the gallbladder originate in the skin. However, the oral cavity, ano-rectalregion, uveal tract, and meninges are all potential sites of primary melanoma.

Different image modalities have been used to evaluate gallbladder lesions, including neoplastic ones Ultra-Sound; US; can help distinguish between malignant lesions and polyps; $94 \%$ of benign lesions have a diameter of less than $1 \mathrm{~cm}$, and $88 \%$ of malignant lesions are larger than $1 \mathrm{~cm}$ across. The presence of flow on color Doppler US images helps exclude the possibility of tumefactive sludge and heightens concern for malignant involvement. Conversely, demonstrating that an intra-luminal mass is mobile by changing the patient's position at US helps confirm that the mass is not a gallbladder neoplasm or polyp. In cases of biliary tree involvement, US also may show intraluminal mural masses and dilatation of the bile duct.

The findings of primary or metastatic gallbladder melanoma on CT scans are similar to those on US images: Focal irregular wall thickening or one or more enhancing intra-luminal polypoid masses can be seen. On magnetic resonance images, findings are similar. Primary \& metastatic melanotic melanomas typically show hyper-intense signal on T1-weighted images and hypo-intense signal on T2-weighted images because of T1-shortening effect of melanin. However, because the amount of melanin within these lesions varies and other factors such as hemorrhage or necrosis may be present, the signal characteristics are inconstant, and a wide range of signal intensities may be seen. Administering gadolinium is not helpful for further characterizing melanotic lesions that display signal hyper-intensity on T1- weighted images. In fact, enhancement of the normal gallbladder mucosa may hinder visualization of these lesions.

Oral cholecystographic, intra-venous cholangiographic, and endoscopic retrograde cholangio-pancreatographic images may depict intra-luminal GB filling defects; however, frequently the gallbladder is not visible, and consequently it is not possible to differentiate melanoma of the gallbladder from chronic a calculous cholecystitis with these techniques. Fl-18 fluorodeoxy glucose positron emission tomography with CT fusion imaging can greatly help correctly identify a metastatic focus of melanoma in the gallbladder.

The differential diagnosis of GB melanoma includes gallbladder adenocarcinoma, which is much more common than gallbladder melanoma and may have a similar appearance 


\section{Cancer Therapy \& Oncology International Journal}

on US and CT images. Gallbladder adenocarcinoma is usually associated with gallstones and is more common in women and older patients. Much rarer than gallbladder adenocarcinoma are unusual epithelial and non-epithelial malignancies as squamous cell carcinoma, carcino-sarcoma, small cell carcinoma, and lymphoma. Malignant melanoma is the most common cause of metastatic tumors of gallbladder. However, other primary tumors, such as renal cell carcinoma, may metastasize to the gallbladder hematogenously. Furthermore, advanced hepatocellular carcinoma can locally extend and invade the gallbladder, and it can even penetrate through the gallbladder wall and produce an endo-luminal mass.

Benign polyps such as cholesterol polyps, inflammatory polyps, and adenomas may manifest as polypoid lesions within the gallbladder, but they tend to be much smaller than malignant lesions. Focal areas of adenomyomatosis, which are also known as adenomyomas, may appear as sessile polypoid lesions. However, additional imaging features of adenomyomas, such as cystic spaces within the gallbladder wall and echogenic foci with ring down or twinkling artifacts on color Doppler US images, usually help distinguish them from neoplasia.

Malignant melanoma is FDG-avid and the sensitivity and specificity of distant metastasis measured by FDG PET/CT is reported to be $92 \%$ and $90 \%$, respectively. Whole body FDG $\mathrm{PET} / \mathrm{CT}$ is cost-effective in the imaging evaluation of patients at high risk for metastatic disease. FDG PET/CT impacts the clinical management of patients with melanoma by detecting unknown metastases prior to planned surgical intervention. PET/CT can also be useful in evaluating treatment response. In fact, FDG PET/CT is evolving as a standard diagnostic imaging tool in patients with melanoma. FDG PET/CT provides more accurate assessment of the extent of disease in-comparison to other conventional imaging techniques, leading to significant alterations in treatment planning. From the referring physician's perspective, FDG PET/CT has a major impact on the management of patients with melanoma by either down-staging or up-staging the disease leading to changes in treatment strategy in up to 53\% of patients.FDG PET/CT offers a diagnostic advantage over other conventional imaging modalities for the detection of metastatic disease; in-particular, odd, unexpected and rare lesions in asymptomatic patients; just like our case. From the literature, the prognosis of metastatic melanoma to the gallbladder is dismal, with a median survival of 6-9 months and long-term disease-free survival is achieved in only $1 \%-2 \%$ of patients.

\section{Once involvement of GB is detected, it is likely that metastases are widespread}

The optimal therapy for metastatic gallbladder melanoma is not definitive; however, treatment options depend on the extension of the disease and on the clinical status of the patient. When metastases are limited to the gallbladder, surgical treatment is indicated to avoid symptoms or tumor complications and can improve prognosis significantly. Nevertheless, even in case of disseminated disease, surgical removal seems to be a worth wise palliative procedure. Chemo-immuno-therapy is a palliative treatment procedure for stage 4 patients. It prolongs disease-free survival, but does not have any effect on 5-year survival rates and the rate of 5-year survivalis approximately $5 \%$. There is a need for further studies about this issue. Current studies propose the use of high-dose interferon. However, there is no immunologic or chemotherapeutic agent available today, which is proven to ensure advantages on survival of metastatic melanoma and despite the advances made in chemotherapy and immunotherapy for metastatic melanoma, the results are still poor and disappointing.

\section{Conclusion}

In general, melanoma is FDG-avid and the sensitivity and specificity of distant metastasis measured by FDGPET/CT is reported to be $92 \%$ and $90 \%$, respectively. Accordingly, FDG $\mathrm{PET} / \mathrm{CT}$ is considered a powerful imaging modality that can afford a crucial role in detection of rare metastatic melanoma lesions and provides more accurate staging information to the therapists' team. Whole body FDG PET/CT is proved to be costeffective in evaluation of patients at high risk for metastatic disease and is also useful in therapy monitoring.

In fact, FDG PET/CT is evolving as a standard diagnostic imaging tool in patients with melanoma as it provides more accurate assessment of the extent of disease and is considered superior to other conventional imaging techniques; in-particular, odd, unexpected and rare lesions in asymptomatic patients; leading to significant alterations in therapy plans in more than $50 \%$ of patients.

\section{Reference}

1. Shimada K, Ichikawa T, Aono G, Oohara T, Abe R, et al. (2010) Malignant melanoma of rare occurrence in the digestive organs; review of the Japanese literature. Geka Jpn J Surg 72: 503-508.

2. Katz SC, Bowne WB, Wolchok JD, Busam KJ, Jaques DP, et al. (2007) Surgical management of melanoma of the gallbladder: a report of 13 cases and review of the literature. Am J Surg 193(3): 493-497.

3. Takada $\mathrm{T}$ (2007) Clinical practice guidelines for management of biliary tract \& ampullary carcinomas. J Hepatobiliary Pancreat Surg 15(1): 1.

4. M Guida, A Cramarossa, A Gentile, M De Lena (2003) Metastatic malignant melanoma of the gallbladder: a case report and review of the literature. Melanoma Research 12(6): 619-625.

5. U Kohler, T Jacobi, G Sebastian, M Nagel (2000) Laparoscopic cholecystectomy in isolated gallbladder metastasis of malignant melanoma. Chirurg 71(12): 1517-1520.

6. DL Bartlett (2000) Gallbladder cancer. Semin Surg Oncol 19(2): 145155.

7. Schwimmer J, Essner R, Patel A, Jahan SA, Shepherd JE, et al. (2000) A review of the literature for whole-body FDG PET in the management of patients with melanoma. Q J Nucl Med 44(2): 153-167.

8. Cellerino P, Corsi F, Morandi E, Foschi D, Trabucchi E (2000) Metastatic melanoma of the gallbladder. Eur J Surg Oncol 26(8): 815-816.

9. Dong XD, De Matos P, Prieto VG, Seigler HF (1999) Melanoma of the gallbladder: are view of cases seen at Duke University Medical Center. Cancer 85(1): 32-39. 


\section{Cancer Therapy \& Oncology International Journal}

10. Blecker D, Abraham S, Furth EE, Kochman ML (1999) Melanoma in the gastrointestinal tract. Am J Gastro enterol 94(12): 3427-3433.

11. BJ Holloway, DM King (1997) Ultrasound diagnosis of metastatic melanoma of the gallbladder. Br J Radiol 70(839): 1122-1125.

12. MH Seelig, K Schonleben (1997) Laparoscopic cholecystectomy for a metastasis of a malignant melanoma in the gallbladder. Zeitschrift fur Gastroenterologie 35(9): 673-675.

13. Manolidis S, Donald PJ (1997) Malignant mucosal melanoma of the head and neck: review of the literature and report of 14 patients. Cancer 80: 1373-1386.

14. Ollila DW, Essner R, Wanek LA, Morton DL (1996) Surgical resection for melanoma metastatic to the gastrointestinal tract. Arch Surg 131(9): 975-980.

15. Isiklar I, Leeds NE, Fuller GN, Kumar AJ (1995) Intracranial metastatic melanoma: cor-relation between MR imaging characteristics and melanin content. AJR Am J Roentgenol 165(6): 1503-1512.

16. Avila NA, Shawker TH, Fraker D (1994) Color-flow Doppler ultrasonography in metastatic melanoma of the gallbladder. J Clin Ultrasound 22(5): 342-347.
17. Balch CM, Houghton AM (1992) Diagnosis of metastatic melanoma at distant sites. Cutaneous melanoma ( ${ }^{\text {nd }}$ edn), Lippincott, Philadelphia, USA, pp. 439-467.

18. Stutte H, Muller PH, D Hoedt B, Stroebel W (1989) Ultrasonographic diagnosis of melanoma metastases in liver, gallbladder, and spleen. J Ultrasound Med 8(10): 541-547.

19. DI Heath and C Womack (1988) Primary malignant melanoma of the gall bladder. Journal of Clinical Pathology 41(10): 1073-1077.

20. N Daunt, DM King (1982) Metastatic melanoma in the biliary tree. British Journal of Radiology, 55(659): 873-874.

21. Backman H (1969) Metastases of malignant melanoma in the gastrointestinal tract. Geriatrics 24: 112-120.

22. Abrams HL, Spiro R, Goldstein N (1950) Metastases in carcinoma; analysis of 1000 autopsied cases. Cancer 3(1): 74-85.

\section{Your next submission with Juniper Publishers} will reach you the below assets

- Quality Editorial service

- Swift Peer Review

- Reprints availability

- E-prints Service

- Manuscript Podcast for convenient understanding

- Global attainment for your research

- Manuscript accessibility in different formats

( Pdf, E-pub, Full Text, Audio)

- Unceasing customer service

Track the below URL for one-step submission https://juniperpublishers.com/online-submission.php 